\title{
Percepción de la cultura de seguridad del paciente en enfermeros de atención primaria
}

\author{
Francisco A. Vega-Ramírez', Javier Giménez Salazar¹, Remedios López-Liria² \\ ${ }^{1}$ Distrito Sanitario Poniente de Almería. El Ejido. España \\ ${ }^{2}$ Departamento de Enfermería, Fisioterapia y Medicina. Centro de Investigación en Salud. \\ Universidad de Almería. Almería. España
}

(Recibido el 22 de Octubre de 2019, Aceptado el 23 de Enero de 2020)

\section{RESUMEN:}

Objetivo: describir la percepción que tienen 201 enfermeros en relación a la seguridad del paciente (SP) en sus centros, valorar cuáles son los puntos fuertes y las áreas de mejora en los Distritos Sanitarios de la provincia de Almería.

Metodología: Se ha utilizado el cuestionario denominado MOSPSC (Medical Office Survey on Patient Safety Culture), traducido, adaptado y validado para profesionales españoles de Atención Primaria. A través del programa estadístico SPSS, se calculó la media y el intervalo de confianza al 95\% de cada una de las dimensiones del cuestionario.

Resultados: se observa una percepción positiva de la SP en casi todas las dimensiones, a excepción de la "comunicación sobre el error", el "seguimiento de la atención a los pacientes", la "comunicación franca" y el "ritmo y carga de trabajo". Uno de los puntos fuertes de los enfermeros es el "trabajo en equipo".

Conclusiones: Los resultados de esta investigación también han destacado "el intercambio de información con otros equipos"; en cambio, entre las áreas de mejora que deberían tenerse en cuenta se halla, "la comunicación franca y sobre el error". Estos resultados deberían ayudar a evaluar la importancia de la SP en las organizaciones, para dialogar sobre los errores y crear un clima de aprendizaje y deseo de mejora en los profesionales que repercuta en la calidad de la atención ofrecida a los usuarios.

Palabras clave: cultura de seguridad; comunicación; errores; atención primaria.

\section{Perception of Patient'S Safety Culture in Primary Care Nurses}

\section{ABSTRACT:}

Aim: to describe the perception of 201 nurses, regarding patient safety in their centers, to determine the strengths and areas of improvement in Health District of Almería. 
Methodology: The Medical Office Survey on Patient Safety Culture questionnaire (MOSPSC) was used translated, adapted and assessed for Spanish primary healthcare proffesionals in Primary Care. Using the statistic program SPSS was calculated the mean and 95\% confidence interval of each of the questionnaire dimensions.

Results: A positive perception was found in the SP for almost all of the questionnaire dimensions, with the exception of "error communication", "patient care follow-up", "clear communication" and "work load and work rhythm". One of the strengths of the nurses is "team work".

Conclusions: the results have also highlighted the "information exchange with other healthcare departments"; on the other hand, among those areas to be improved we find "clear communication and error communication". The results of these questionnaires may assist in evaluating the importance of patient safety in the organizations, in order to create dialogue on the errors and create a climate of learning and a desire to improve in healthcare professionals, with positive repercussions on the quality of care offered to users.

Keywords: Patient'S Safety Culture; Communication; Errors; Primary Care.

Correspondencia: Remedios López-Liria. Edificio Ciencias de la Salud. Ctra del Sacramento S/N. La Cañada de San Urbano, Almería 04120.

\section{Introducción}

Una cultura de seguridad es "aquella en la que las organizaciones, prácticas, equipos y personas tienen una consciencia constante y activa del potencial de las cosas que pueden fallar" (Ministerio de Sanidad y Consumo, 2009, p. 30). En las instituciones sanitarias, también se ha definido como los esfuerzos integrados de individuos y grupos para reducir el daño al paciente a través de las interacciones, actitudes y compresión de los procesos de seguridad (Cooper, 2000; Yari, Naseri, Akbari, Shahsavari y Akbari, 2019).

La investigación sobre seguridad del paciente (SP) es relativamente joven, en 1999 en Estados Unidos, la agencia para la investigación y la calidad del cuidado de la salud (en inglés, Agency for Healthcare Research and Quality, AHRQ) definió las prácticas de SP como "las intervenciones, estrategias o aproximaciones para prevenir o mitigar consecuencias inintencionadas derivadas de los cuidados de salud y para mejorar la seguridad del cuidado para los pacientes" (Shekelle et al., 2010, p.14).

En España, la práctica sanitaria en Atención Primaria (AP) es segura. Un importante estudio identificó 11.20 eventos adversos por cada mil visitas, estudiando 48 centros de AP de 16 Comunidades Autónomas (Aranaz-Andrés et al., 2012). Numerosos factores pueden contribuir al desarrollo de estos incidentes de seguridad (es decir, incidentes que no llegan al paciente, incidentes sin daños y eventos adversos), factores relacionados con la organización, normas explícitas e implícitas, los recursos, errores humanos o las características de los pacientes (Borrell, 2007; Reyes-Alcazar, Cambil y Herrera-Usagre, 2013). Algunos estudios sobre salud y seguridad señalan que de un 85 a un 98 por ciento de los comportamientos que llevan a incidentes de seguridad se deben a una inadecuada cultura de seguridad (Chen, McCabe y Hyatt, 2017; Jafari, Gharari, Kalantari, Omidi, Ghaffari y Fardi, 2015).

La asistencia sanitaria siempre ha implicado riesgos, que se pueden minimizar o reducir analizando y abordando la causa o raíz de estos incidentes en la seguridad del paciente. El modo

(C) Psy, Soc, \& Educ, 2020, Vol. 12(2) 
de afrontar los eventos adversos en el Sistema Nacional de Salud (SNS) debería interpretarse como oportunidades de mejora en las intervenciones de los profesionales, servir para prevenir futuros daños, y no señalarlos como errores humanos (Astier-Peña, Torijano-Casalengua y Olivera-Cañadas, 2016). Se ha indicado, que la sensación de no saber cómo van a ser tratados los sucesos adversos y/o la percepción punitiva que puedan recibir los profesionales sanitarios, ofrecen una cierta pérdida de confianza en los aspectos de seguridad (Tomas, Chánovas, Roqueta y Toranzo, 2012).

Existe una clara necesidad de desarrollar una cultura de seguridad que por una parte acepte los riesgos inherentes a la asistencia sanitaria y, por otra, hable abiertamente de incidentes o errores, que en ocasiones plantean consecuencias graves, pérdida de reputación y desconfianza. La evidencia demuestra que si la cultura de una organización es consciente de la seguridad, y las personas son estimuladas a hablar abiertamente sobre errores e incidentes en su lugar de trabajo, la seguridad del paciente y la atención al mismo se ven sin duda mejoradas (Ministerio de Sanidad y Consumo, 2009). En una encuesta a 195 directivos, se concluye que la mayoría de las instituciones (el $45.20 \%$ de AP, y el $54.80 \%$ de hospitales) no tenían un plan de crisis para salvaguardar su reputación tras un evento adverso (Mira, Carrillo y Lorenzo, 2017).

Se plantea la necesidad de obtener datos concretos que ayuden a evitar incidentes de seguridad que afecten a los usuarios, cuestión que se caracteriza por mermar su calidad de vida, evoluciones más prolongadas de la enfermedad o incluso el coste de vidas y unos gastos económicos relevantes en el entorno familiar, comunitario y social que desembocan en una pérdida de la relación coste-efectividad, comprometiendo la sostenibilidad del SNS público (Consejería de Salud, 2011; Contel, Muntané y Camp, 2012; Limón, Blay y Ledesma, 2015; Martín Lesende, 2013; Morales Asencio, 2013; Pericas, González, de Pedro, Morales, y Bennasar, 2014; Repu1lo, 2012; Terraza, Ingrid, Lorenzo, Luisa y Navarrete, 2006). Esto podría evitarse mediante la promoción de una cultura de seguridad, una formación y aprendizaje tras identificar áreas de mejora, que permitan elaborar protocolos de prevención y decálogos de seguridad en los cuidados que son aplicados (de Andrés Gimeno, Salazar, Ferrer, Revuelta, Ayuso y González, 2014). Por lo tanto es necesario identificar los puntos críticos y promover nuevas líneas de actuación que contribuyan a convertir la seguridad dentro del contexto sanitario, en un valor presente en cada práctica, cada momento y para cada usuario, donde los profesionales actúen de forma proactiva en el rediseño de sus procesos asistenciales para evitar nuevos incidentes (Camargo Tobias, Queiroz-Bezerra, Silvestre-Branquinho, de Camargo Silva, 2014).

El objetivo de esta investigación ha sido describir la percepción que tienen los enfermeros sobre las actividades que se desarrollan en relación a la seguridad del paciente en sus centros, valorar cuáles son los puntos fuertes y las áreas de mejora en los Distritos Sanitarios de la provincia de Almería.

\section{Método}

Estudio descriptivo transversal en 201 enfermeros que desarrollan su labor en alguno de los Distritos Sanitarios de la provincia de Almería (Distrito Sanitario Poniente, Distrito Sanitario Almería o el Área Sanitaria Norte). Se ha utilizado el cuestionario denominado MOSPSC ("Medical Office Survey on Patient Safety Culture," 2011), traducido, adaptado y validado para profe- 
sionales españoles de AP, para valorar la cultura de SP (Torijano-Casalengua, Olivera-Cañadas, Astier-Peña, Maderuelo-Fernández y Silvestre-Busto, 2013). Para dicha adaptación se aplicó a 185 profesionales, concluyéndose que era fiable y útil. El coeficiente alfa de Cronbach para el total de la escala fue de 0,96 (Torijano-Casalengua et al., 2013).

Las variables estudiadas han sido la edad, el sexo, el puesto que ocupa, número de tarjetas sanitarias adscritas, el número de años en activo, su horario, etc.; así como las variables sobre las dimensiones de la cultura de seguridad que registra el cuestionario (véase la figura 1). Finalmente se elaboró una pregunta abierta construida "ad hoc" para solicitar ideas destinadas a mejorar esta seguridad: acciones que redundarían en un mayor impacto sobre la cultura de SP.

A través del programa estadístico SPSS versión 22, se calculó la media y el intervalo de confianza al $95 \%$ de cada una de las dimensiones del cuestionario para las respuestas de los enfermeros, considerándose una puntuación $>3$ percepción positiva y claramente positiva (puntuación>4) (Ministerio de Sanidad Servicios Sociales e Igualdad, 2014).

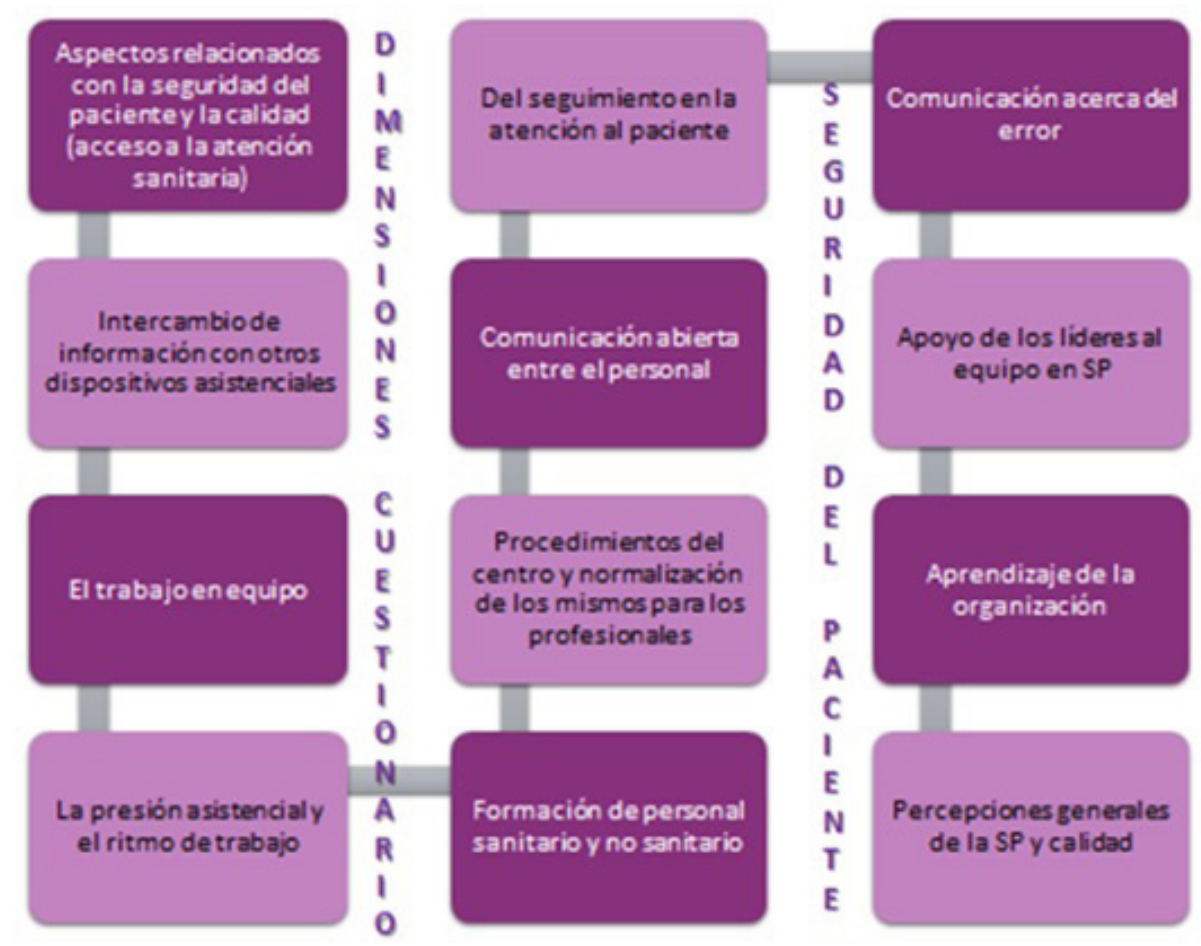

Figura 1. Principales dimensiones del cuestionario de Seguridad del Paciente.

\section{Resultados}

La edad media de los enfermeros que respondieron fue de 48 años y el $73.61 \%$ mujeres. $\mathrm{Su}$ actividad asistencial mostró una media de 23 años. El número de tarjetas sanitarias adscritas a este personal fue de 1718 de media. El 10.45\% de las personas que han respondido este cuestionario ocupaban un puesto de gestión, responsabilidad o liderazgo. El 51.05\% tenía un turno rotatorio, frente al $47.55 \%$ que trabaja de mañanas, un $1.39 \%$ de tardes. Un $68.28 \%$ realizaban guardias. El $69.41 \%$ fue personal fijo, el $25.69 \%$ eventual y el $4.86 \%$ interino. 
En la tabla 1 se resumen los principales hallazgos en relación a las 15 dimensiones que miden la cultura de seguridad del paciente en estos enfermeros de AP, además de la puntuación global sobre la calidad (media 3.40) y seguridad del paciente (2.97).

Tabla 1. Dimensiones cuestionario seguridad del paciente en enfermeros

Dimensión (Preguntas que la integran)

1. Aspectos relacionados con la seguridad del paciente y calidad (A1 A2 A3 A4 A5 A6 A7 A8 A9)

2. Intercambio de información con otros dispositivos asistenciales (B1 B2 B3 B4)

3. Trabajo en equipo (C1 C2 C5 C13)

4. Ritmo y carga de trabajo (C3 C6 C11 C14)

5. Formación del personal no sanitario (C4 - C7 C10)

6. Formación del personal sanitario (C16 C17 C18)

7. Procedimientos establecidos en el centro (C8 C9 C12 C15)

8. Procedimientos establecidos en el centro. Profesionales sanitarios (C8 C9 C12 C19)

9. Comunicación Franca (D1 D2 D4 D10)

10. Seguimiento de la atención a los pacientes (D3 D5 D6 D9)

11. Comunicación sobre el error (D7 D8 D11 D12)

12. Comunicación sobre el error. Profesionales sanitarios D8 D11 D13 D14)

13. Apoyo de los responsables del centro a la seguridad del paciente (E1 E2 E3 E4)

14. Aprendizaje Organizacional (F1 F5 F7)

15. Percepciones generales sobre la seguridad del paciente y la calidad (F2 F3 F4 F6)

16. Puntuaciones globales sobre la calidad (G1 G2 G3 G4 G5)

17. Puntuaciones globales en seguridad del paciente (G2)
Total Media

(IC95\%)

$4.13(4.04-4.22)$

$4.18(4.02-4.33)$

$4.01(3.91-4.10)$

$2.78(2.65-2.91)$

$3.49(3.32-3.66)$

$3.53(3.40-3.66)$

$3.44(3.30-3.58)$

$3.54(3.43-3.65)$

$2.24(2.10-2.37)$

$2.08(1.92-2.24)$

$2.19(2.02-2.36)$

$2.14(2.01-2.27)$

$3.42(3.25-3.59)$

$3.74(3.61-3.87)$

$3.68(3.56-3.80)$

$3.40(3.27-3.53)$

$2.97(2.80-3.14)$

A continuación se presentan las principales áreas clave señaladas por los profesionales en las que las organizaciones y equipos de AP deberían trabajar para salvaguardar esta seguridad, produciendo un mayor impacto en la cultura de seguridad del paciente:

- Realización de sesiones informativas y formativas sobre SP con instructores bien formados. Visitas directivas regulares a toda la organización, reuniendo al personal y en ocasiones a pacientes y familiares, para tratar específicamente temas de seguridad que les preocupan o errores frecuentes que vivencian.

- Deberían existir mecanismos que incentiven al personal para sugerir ideas sobre cómo mejorar el desempeño de su trabajo, fomento de discusiones sobre incidentes de SP que han sucedido, fueron evitados o casi sucedieron ("casi errores") en su puesto, sin que suponga "miedo" a repercusiones negativas o sean vistas como errores humanos fruto de nuestras limitaciones. 
- Adecuados medios de comunicación y reacción para que el personal comprenda su contribución a la seguridad y sea animado a participar (tipos de incidentes, causas, condiciones latentes y evitabilidad de los eventos adversos). Esta comunicación debería ser como previamente se ha señalado en otros estudios, intencional, planificada y centrada en objetivos relacionados con la estrategia de seguridad del centro (Mira, et al., 2017).

- Para implantar prácticas seguras en el SNS, sería necesario consensuar una historia clínica informatizada que además esté unificada, integrando a los distintos agentes y sistemas que participan a la hora del diagnóstico, la prescripción y el seguimiento de los cuidados (especialistas, médicos, enfermeros, fisioterapeutas, etc.). Así se conseguiría la identificación unívoca del paciente y el acceso en tiempo real a la información clínica de muestras, resultados de técnicas, volantes de derivación, sistemas de alertas ante resultados anómalos de pruebas, uso seguro y confidencial de la información (Astier-Peña et al., 2016).

- También se indicó la necesidad de proporcionar planes de recepción de nuevos profesionales en los centros donde los "más antiguos" tutoricen a los profesionales "nóveles" durante un tiempo (la inexperiencia, especialmente si se combina con la falta de supervisión son factores de riesgo); la corresponsabilidad de los profesionales en el seguimiento de los pacientes; la formación en el manejo de pacientes crónicos, pluripatológicos y polimedicados, así como instrucción en los aparatos y nuevas tecnologías incorporadas en los centros de salud; fomentar sesiones formativas de análisis de los incidentes (grupos focales y de expertos); promover unidades funcionales de riesgos y la presencia de un referente en el equipo de SP al que dirigirse ante estas situaciones (Astier-Peña et al., 2016).

- Y no menos importante resultó para los profesionales indicar, que es preciso favorecer la participación y comunicación con los pacientes y sus familiares, para garantizar una mayor apertura e integración del sistema de salud en el entorno comunitario. A pacientes y cuidadores se les debe implicar en materia de seguridad, ofreciéndoles mensajes claros para que conozcan la medicación que toman, su autocuidado, que nos alerten de cualquier situación que pueda comprometer su salud o seguridad, que hagan preguntas, etc.

\section{Discusión}

Los equipos de AP deben adaptarse a las necesidades de las personas, lo que exige un análisis continuo, objetivo y crítico de la efectividad de sus actuaciones, mediante una reconsideración flexible y de mentalidad abierta sobre sus prestaciones, desterrando las prácticas ineficientes y que ponen en riesgo la seguridad del paciente (Limón et al., 2015; Terraza et al., 2006).

En AP hay que afrontar una enorme agenda y una complejidad en el volumen de trabajo que puede tener un impacto sobre la cultura de seguridad de esta organización, aumentando el riesgo de daño involuntario al paciente. Factores que tienen incidencia en su desarrollo son por ejemplo, los cambios en los planes de trabajo y gestión de pacientes, los avances en la tecnología, el desplazamiento de un gran volumen de trabajo desde Atención Especializada (AE) a AP, constantes cambios en el personal, crecientes necesidades de atención sanitaria y social de los pacientes crónicos (incluyendo tratamientos farmacológicos más complejos y estándares clínicos nacionales muy exigentes), así como un mayor examen externo (Ministerio de Sanidad y Consumo, 2009). 
En los resultados de esta investigación se ha observado en general una percepción positiva de la SP en casi todas las dimensiones del cuestionario, a excepción de: el "seguimiento de la atención a los pacientes", la "comunicación sobre el error", el "ritmo y carga de trabajo" y la "comunicación franca”. En el estudio de Torijano-Casalengua et al., las dimensiones que obtuvieron una peor respuesta fueron los "aspectos relacionados con la SP y la calidad, junto a su percepción general", el "intercambio de información con otros dispositivos asistenciales" y el "aprendizaje organizacional". Otra investigación a nivel nacional nos indica que la dimensión "ritmo y carga de trabajo" fue la peor valorada (Ministerio de Sanidad Servicios Sociales e Igualdad, 2014). Respecto a la percepción en SP que tienen los profesionales del Distrito Sanitario Poniente en Almería, en un estudio de 2017 se indica que las dimensiones con una percepción claramente positiva fueron los "aspectos relacionados con la seguridad del paciente y calidad", el "intercambio de información con otros dispositivos asistenciales" y el "trabajo en equipo" y las dimensiones que obtuvieron una peor respuesta fueron la "comunicación sobre el error" y el "seguimiento de la atención a los pacientes” (López-Liria, Rocamora-Pérez, Aguilar-Parra, Vargas-Muñoz, DíazLópez, Padilla-Góngora, 2017).

El estudio de Martínez Ques y colaboradores (2010) también tuvo como objetivo evaluar las barreras que los profesionales de enfermería detectan en su práctica clínica para el desarrollo de una cultura de seguridad en la práctica cotidiana, concluyendo que las debilidades giran en torno a la profesión, organización e infraestructura, indicadores, comunicación y cultura de seguridad, así como la formación en seguridad (Martínez Ques, Hueso Montoro, Gálvez González, 2010).

Uno de los puntos fuertes de los enfermeros de AP en la provincia de Almería es el "trabajo en equipo" como previamente se ha confirmado en otras investigaciones nacionales (López-Liria et al., 2017; Ministerio de Sanidad Servicios Sociales e Igualdad, 2014). Entre las características que conforman el trabajo en equipo se debe compartir un objetivo, poseer sistemas de comunicación y evaluación comunes, ser un modelo de trabajo transdisciplinar, con una comunicación clara, veraz, fluida y empática, lo que genera mejores resultados clínicos e incrementa la satisfacción profesional (Catalán-Matamoros, Muñoz-Cruzado y Fuentes Hervías, 2010; Escarrill, Cleries y Sarrado, 2015).

Los resultados de este trabajo también han destacado "el intercambio de información con otros equipos"; en cambio, entre las áreas de mejora que deberían tenerse en cuenta se halla, "la comunicación franca y sobre el error". Se ha descrito que las organizaciones con una cultura de seguridad positiva se basan en la comunicación y la confianza mutua, son capaces de reconocer los errores, aprender de ellos y actuar para su rectificación con acciones preventivas (Ministerio de Sanidad Servicios Sociales e Igualdad, 2014). También se ha señalado en diversos estudios, que la comunicación influye en el grado de satisfacción de los pacientes, pues al percibir un ambiente de confianza y ser escuchados, propicia un mejor conocimiento de su enfermedad y seguimiento de las recomendaciones realizadas por los profesionales sanitarios de referencia; el uso de una comunicación eficaz facilitaría la promoción de la salud y la prevención de la enfermedad; cuando ésta falla, se producen la mayoría de las reclamaciones de los pacientes (Catalán-Matamoros, Muñoz-Cruzado y Fuentes Hervías, 2010; Díaz-Sáez, Catalán-Matamoros, Fernández-Martínez y Granados-Gámez, 2011). Muchos de los problemas o incidentes sanitarios son producidos por la falta de comunicación. Algunos profesionales identifican como principales cualidades de los gerentes exitosos y por tanto la gestión excelente de esas unidades: el compro- 
miso de la dirección por la seguridad, la preocupación por los trabajadores, la congruencia entre los mensajes hablados y la práctica, el profesionalismo y las habilidades de comunicación (Vega Ramírez, Catalán Matamoros, López Liria, 2010).

España dispone uno de los diez mejores modelos de AP del mundo (Astier-Peña et al., 2016), pero la falta de inversión en este servicio ofrecido por el SNS afecta desfavorablemente a la seguridad del paciente, como se ve reflejado en la dimensión "ritmo y carga de trabajo" en todos los estudios evaluados (López-Liria et al., 2017; Ministerio de Sanidad, Servicios Sociales e Igualdad, 2014; Pozo y Padilla, 2013; Torijano-Casalengua, et al., 2013). Cuando existe un elevado ritmo y carga de trabajo, puede afectar la fatiga o se pueden tomar decisiones apresuradamente que entran en conflicto con la SP. Otra cuestión que interfiere es cuando el propio sistema está mal definido: existe una formación o capacitación deficiente de los profesionales; un desajuste entre la capacidad y los recursos disponibles junto a la excesiva demanda; una retroalimentación deficiente o falta de comunicación; una definición inadecuada de roles y responsabilidades en el lugar de trabajo.

Un estudio sobre SP en urgencias y emergencias ha señalado que entre las condiciones latentes de riesgos se hallan los problemas de comunicación entre profesional-paciente, profesional-profesional o en los cambios de turno o guardia; la motivación y experiencia; condiciones de trabajo como la escasa información sobre el paciente o dificultad de acceso a la historia clínica, interrupciones y distracciones, sobrecarga asistencial o carteras de servicios heterogéneas (Tomas, Chánovas, Roqueta y Toranzo, 2012).

Una revisión de la literatura identificaba 6 categorías de relevancia en los eventos adversos experimentados por los pacientes de atención domiciliaria (AD): relacionados con medicamentos, relacionados con catéter, con el uso de tecnología, infecciones asociadas a sondaje vesical, heridas, úlceras por presión (9.11\%) y caídas (en un 30\% de los mayores de 65 años) (Masotti, 2010; Ministerio de Sanidad y Política Social, 2009). Las tasas globales de eventos adversos publicadas han variado entre el 3.50 y el $15.10 \%$, por lo que se puede concluir que se producen con relativa frecuencia y con cierto grado de previsibilidad para las categorías identificadas en $\mathrm{AD}$, además de que podrían ser evitables en gran medida (Masotti, McColl y Green, 2010). Existe evidencia científica de que la valoración de los riesgos y la modificación de éstos tiene efectos sobre la incidencia de las caídas, del mismo modo que la utilización de superficies especiales para el manejo de la presión disminuye la aparición de UPP. Debido a estas cifras, se precisan enfoques dirigidos a prevenir o reducir el impacto de estos eventos adversos mediante cambios en los diferentes niveles de participación (pacientes, cuidadores, profesionales sanitarios, entorno físico del hogar, organización sanitaria, etc.) (Consejería de Salud, 2010), ya que se identifican como principales causas de estos eventos una deficiente comunicación entre individuos (incluyendo la educación ofrecida al paciente y cuidador) y entre los niveles participantes en el proceso de cuidado (coordinación asistencial) (Ministerio de Sanidad y Consumo, 2006). Según el estudio EVADUR (Tomás, 2010), los factores causales más frecuentes responsables de incidentes en urgencias son: el manejo inadecuado del paciente, retraso o error en el diagnóstico, la reacción adversa a la medicación u omisión de dosis, el manejo inadecuado de la técnica, comunicación médico-paciente, médico-enfermera o enfermero-paciente, inadecuado manejo de signos de alerta, retraso en la consulta especializada.

En 2004, ya la agencia AHRQ indicaba algunos atributos culturales de la organización que contribuían a la seguridad del paciente, como el trabajo en equipo, el apoyo o soporte de los lí- 
deres y la comunicación (Camargo Tobias, Queiroz-Bezerra, Silvestre-Branquinho, de Camargo Silva, 2014; Sorra y Nieva, 2004).

Además, se observa que se precisan iniciativas en el sistema sanitario como la implementación de una definición estándar para los diferentes tipos de eventos adversos específicos; sistemas de recogida y notificación adecuados y consensuados; y sistemas de vigilancia de estos eventos para la mejora y el aprendizaje continuo, indicadores que nos permitan mantener periódicamente una fotografía sobre los principales problemas y su evolución, así como la necesidad de diseño de nuevas actividades (Consejería de Igualdad, Salud y Políticas Sociales, 2014; Tomas, Chánovas, Roqueta y Toranzo, 2012).

Entre las limitaciones de este estudio se encuentran que debe de hacerse con precaución su generalización a otros ámbitos del sistema sanitario, ya que este trabajo se realizó tan solo en una provincia en AP. Además no se utilizó un muestreo aleatorio, lo que pudo introducir un sesgo de selección y los resultados se han basado en la información aportada por los enfermeros, sin contrastarse por otros medios. Como ya han indicado previamente otros autores, la mejora de la seguridad quizás deba contemplar diferentes estrategias, según el ámbito del que se trate (Tomas, Chánovas, Roqueta y Toranzo, 2012).

Se evidencia en este trabajo que se necesita una definición normalizada de las actuaciones realizadas en el ámbito de AP respecto a la seguridad del paciente, para ello son necesarios más estudios de cohorte prospectivos que describan las características de los pacientes, los servicios que le son ofrecidos, estimaciones de los riesgos para su salud y se deberían llevar a cabo estudios de intervención para reducir los mismos (Morales Asencio, 2011). Como ya han señalado previamente otros autores internacionales, urge evaluar y difundir estudios basados en la evidencia, con recomendaciones que promuevan los resultados y las investigaciones en seguridad del paciente (Shekelle et al., 2011); diversas revisiones sistemáticas han indicado la necesidad de la opinión de los profesionales para conocer y evaluar los obstáculos y los condicionantes de su práctica clínica, así como los elementos positivos y negativos que emergen del sistema y que apelan a un cambio organizacional para obtener una verdadera cultura de seguridad (Camargo Tobias, Queiroz-Bezerra, Silvestre-Branquinho, de Camargo Silva, 2014) .

\section{Conclusiones}

Abordar la seguridad del paciente conjuntamente y de modo sistemático puede tener un impacto positivo en la calidad y eficacia de toda la asistencia sanitaria. Aunque entre las dimensiones con una percepción claramente positiva de los enfermeros del Distrito Sanitario han destacado los "aspectos relacionados con la seguridad del paciente y calidad" o "el intercambio de información con otros dispositivos asistenciales", las que obtuvieron una peor respuesta fue la "comunicación sobre el error", el "seguimiento de la atención a los pacientes", el "ritmo y carga de trabajo" y la "comunicación franca".

Los resultados de estos cuestionarios deberían ayudar a evaluar la importancia de la seguridad del paciente en las organizaciones, para dialogar sobre los errores y crear un clima de aprendizaje y deseo de mejora de directivos y profesionales, que repercuta en la calidad de la atención ofrecida a los usuarios. 


\section{Agradecimientos}

Realizamos un expreso agradecimiento tanto a los profesionales sanitarios participantes, como a la dirección de Atención Primaria de cada uno de los Distritos que facilitó la recogida de información. Algunos de estos datos han formado parte del trabajo inédito premiado en el XXII Certamen Nacional de "Enfermería de Jaén" y el XI Certamen Nacional de investigación de enfermería "José López Barneo".

\section{Referencias bibliográficas}

Aranaz-Andrés, J.M., Aibar, C., Limón, R., Mira, J.J., Vitaller, J., Agra, Y., et al (2012). A study of the prevalence of adverse events in primary healthcare in Spain. European Journal Public Health. 22(6), 921-925.

Astier-Peña, M.P., Torijano-Casalengua, M.L. y Olivera-Cañadas, G. (2016). Prioridades en seguridad del paciente en Atención Primaria. Atención Primaria, 48(1), 3-7.

Borrell Carrió, F. (2007). Previsión de esfuerzo. Atención Primaria [Internet], 39(10), 569-571.

Camargo Tobias, G., Queiroz-Bezerra, A.L., Silvestre-Branquinho, N.C., de Camargo Silva, A.E.B. (2014). Cultura de la seguridad del paciente en la atención sanitaria: un estudio bibliométrico. Enfermería Global, 33, 336-349.

Catalán-Matamoros, D., Muñoz-Cruzado y Barba, M., Fuentes Hervías, M.T. (2010). Técnicas de comunicación para la prevención y el control de enfermedades. Revista Española de Comunicación en Salud, 1(1), 50-65.

Chen, Y., McCabe, B., y Hyatt, D. (2017). Impact of individual resilience and safety climate on safety performance and psychological stress of construction workers: A case study of the Ontario construction industry. Journal of Safety Research, 61, 167-76.

Consejería de Salud. (2010). Portal de Igualdad, Salud y Políticas Sociales. Plan de Calidad del Sistema Sanitario Público de Andalucía. 2010-2014. Junta de Andalucía. Disponible en: http://www.juntadeandalucia.es/salud/channels/temas/temas_es/P_2_ANDALUCIA_EN_ SALUD_PLANES_Y_ESTRATEGIAS/II_plan_calidad/plan_calidad_2010

Consejería de Salud (2011). Desarrollo competencial en el modelo de Gestión de Casos del SSPA. Estrategia de Cuidados de Andalucía. Consejería de Salud. 2011. Disponible en: http://www.juntadeandalucia.es/servicioandaluzdesalud/principal/documentosAcc. asp?pagina=pr_desa_Innovacion 14 .

Contel, J.C., Muntané, B. y Camp, L. (2012). La atención al paciente crónico en situación de complejidad: el reto de construir un escenario de atención integrada. Atención Primaria, 44(2), 107-113.

Cooper, M. (2000). Towards a model of safety culture. Safety Science, 36, 36-111.

Consejería de Igualdad, Salud y Políticas Sociales. (2014). Evaluación de la estrategia para la Seguridad del Paciente del SSPA. Informe Global. Junta de Andalucía. Observatorio para la seguridad del paciente. Enero 2014.

de Andrés Gimeno, B., Salazar de la Guerra, R.M., Ferrer Arnedo, C., Revuelta Zamorano, M., Ayuso Murillo, D., y González Soria, J. (2014). Una aproximación al benchmarking de indicadores de cuidados. Aprendiendo para mejorar la seguridad del paciente. Revista de Calidad Asistencial [Internet], 29(4), 212-219. 
Díaz-Sáez, J., Catalán-Matamoros, D., Fernández-Martínez, M.M. y Granados-Gámez, G. (2011). La comunicación y la satisfacción de las primíparas en un servicio público de salud. Gaceta Sanitaria, 25(6), 483-489.

Escarrabill, J., Clèries, X., y Sarrado, J. (2015). Competencias transversales de los profesionales que facilitan atención domiciliaria a pacientes crónicos. Atención Primaria [Internet], 47(2), 75-82. Doi: 10.1016/j.aprim.2014.02.009

Jafari, M.J., Gharari M., Kalantari, S., Omidi, L., Ghaffari, M. y Fardi, G.R.A. (2015). The influence of safety training on improvement in safety climate in construction sites of a firm. Safety Promotion and Injury Prevention, 2, 257-64.

Kasatpibal, N., Senaratana, W., Chitreecheur, J., Chotirosniramit, N., Pakvipas, P., Junthasopeepun, P. (2012). Implementation of the World Health Organization surgical safety checklist at a university hospital in Thailand. Surg Infect (Larchmt), 13(1), 50-6.

Limón, E., Blay, C., y Ledesma, A. (2015) Atención Primaria Las necesidades poblacionales, una llamada a la transformación de la atención primaria. Population needs, a call for changes in Primary Care. Atención Primaria [Internet], 47(2), 73-74

López-Liria, R., Rocamora-Pérez, P., Aguilar-Parra, MJ, Vargas-Muñoz, M.E., Díaz-López, M.P., y Padilla-Góngora, D. (2017). Evaluation in primary care professionals: The patient's safety culture. Procedia Social Behavioral Science, 237, 1272-1276. Doi: 10.1016/j. sbspro.2017.02.209.

Martín Lesende, I. (2013). Abordaje integral del paciente pluripatológico en atención primaria. Tendencia necesitada de hechos. Atención Primaria, 45 (4), 181-183.

Martínez Ques, A.A., Hueso Montoro, C., Gálvez González, M. (2010). Fortalezas y amenazas en torno a la seguridad del paciente según la opinión de los profesionales de enfermería. Revista Latino-Americana Enfermagen, 18(3), 42-49.

Masotti, P., McColl, M.A. y Green, M. (2010). Adverse events experienced by homecare patients: a scoping review of the literature. International Journal for Quality in Health Care, 22(2):115-125.

Ministerio de Sanidad y Política Social. (2009). Informe Plan de Calidad para el Sistema Nacional de Salud 2006-2010. Balance de actividades y acciones previstas. Madrid: Ministerio de Sanidad y Política Social.

Ministerio de Sanidad y Consumo (2009). National Patient Safety Agency. "Siete pasos para la seguridad del paciente en Atención Primaria" [Internet]. Madrid. Salud Pública de CSN, editor. Ministerio de Sanidad y consumo. Pp. 177. Disponible en: http://www. calidadasistencial.es/images/gestion_soc/documentos/236.pdf

Ministerio de Sanidad y Consumo. (2006). Agencia Nacional para la Seguridad del Paciente (NPSA)-Sistema Nacional de Salud, Reino Unido, La seguridad del paciente en siete pasos, Agencia de Calidad, Ministerio de Sanidad y Consumo, Madrid.

Ministerio de Sanidad Servicios Sociales e Igualdad. (2014). Análisis de la cultura de la seguridad del paciente de los profesionales de la Atención Primaria del Sistema Nacional de Salud. Informes. Madrid: Ministerio de Sanidad SSEISNDS, editor. Pp. 116.

Mira, J.J, Carrillo, I., Lorenzo, S. (2017). Qué hacen los hospitales y la atención primaria para mitigar el impacto social de los eventos adversos graves. Gaceta Sanitaria, 31, 150-153. 
Morales Asencio, J.M. (2011). La atención sanitaria basada en la evidencia necesita buenos modelos conceptuales para su implementación. Gestión Clínica y Sanitaria, 12(4), 128.

Morales Asencio, J.M. (2013). La gestión de casos y la cronicidad compleja: conceptos, modelos, evidencias e incertidumbres. Enfermería Clínica. Doi: 10.1016/j.enfcli.2013.10.002.

Pericas Beltrán, J., González Torrente, S., de Pedro Gómez, J.E., Morales Asencio, J.M., y Bennasar Veny, M. (2014). Perception of Spanish primary healthcare nurses about evidencebased clinical practice: A qualitative focus group study. International Nursing Review. Doi: 10.1111/inr.12075

Pozo Muñoz, F., y Padilla Marín, V. (2013). Evaluación de la cultura de seguridad del paciente en el ámbito de un área sanitaria. Revista Calidad Asistencial, 28(6), 329-336.

Repullo, J.R. (2012). El Sistema Nacional de Salud en tiempos de crisis: sin primaria no es posible. Atención Primaria, 8(7), 362-363.

Reyes-Alcazar, V., Cambil, J. y Herrera-Usagre, M. (2013). Revisión sistemática sobre recomendaciones de seguridad del paciente para centros sociosanitarios. Medicina Clínica (Barcelona), 141(9), 397-405.

Shekelle, P.G., Pronovost, P.J., Wachter, R.M., Taylor, S.L., Dy, S., Foy, R., et al., and the PSP Technical Expert Panel (2010). Assessing the Evidence for Context-Sensitive Effectiveness and Safety of Patient Safety Practices: Developing Criteria (Prepared under Contract No. HHSA-290-2009-10001C). AHRQ Publication No. 11-0006-EF. Rockville, MD: Agency for Healthcare Research and Quality.

Shekelle, P.G., Pronovost, P.J., Wachter, R.M., Taylor, S.L., Dy, S.M., Foy, R., et al. (2011). Advancing the Science of Patient Safety. Annals Internal Medicine, 154,693-696.

Sorra, J., Nieva, V.F. (2004). Hospital Survey on Patient Safety Culture. AHRQ Publication n. 04-0041. Rockville, MD: Agency for Healthcare Research and Quality.

Terraza, R., Ingrid, N., Lorenzo, V., Luisa, M., y Navarrete, V. (2006). La coordinación entre niveles asistenciales: una sistematización de sus instrumentos y medidas. Gaceta Sanitaria, 20, 485-95.

Tomás, S., Chánovas, M., Roqueta, F., Toranzo T. (2012). La seguridad del paciente en urgencias y emergencias: balance de cuatro años del Programa SEMES-Seguridad Paciente. Emergencias, 24, 225-233.

Tomás S, Chanovas M, Roqueta F, Alcaraz J, Toranzo T y Grupo de trabajo EVADUR - SEMES. (2010). EVADUR: eventos adversos ligados a la asistencia en los servicios de urgencias de hospitales españoles. Emergencias, 22, 415-28.

Vega Ramírez, F.A., Catalán Matamoros, D., López Liria, R. (2010). El rol de las campañas de comunicación en la promoción de la salud y la prevención de lesiones en salud laboral. Revista Española de Comunicación en Salud, 1(2), 137-154.

Torijano-Casalengua, M.L., Olivera-Cañadas, G., Astier-Peña, M.P., Maderuelo-Fernández, J.Á., y Silvestre-Busto, C. (2013). Validación de un cuestionario para evaluar la cultura de seguridad del paciente de los profesionales de atención primaria en España. Atención Primaria, 45, 21-37.

Yari, S., Naseri, M.H., Akbari, H., Shahsavari, S., y Akbari, H. (2019). Interaction of Safety Climate and Safety Culture: A Model for Cancer Treatment Centers. Asian Pacific Journal of Cancer Prevention, 20 (3), 961-969. Doi:10.31557/APJCP.2019.20.3.961. 